\title{
SÉRIES TEMPORAIS DE EVI DO MODIS PARA O MAPEAMENTO DE USO E COBERTURA VEGETAL DO OESTE DA BAHIA
}

Temporal series of EVI from MODIS sensor for land use and land cover mapping of western Bahia

\author{
ELANE FIÚZA BORGES ${ }^{1,2}$ \\ EDSON EYJI SANO ${ }^{1,3}$
}

${ }^{1}$ Universidade de Brasília - UnB/IG

Campus Universitário Darcy Ribeiro, CEP: 70919-970 Brasília/DF/Brasil

${ }^{2}$ Universidade Estadual de Feira de Santana - UEFS/DCHF

Av. Transnordestina, CEP: 44036-900 Feira de Santana/BA/Brasil

${ }^{3}$ Empresa Brasileira de Pesquisa Agropecuária - Embrapa Cerrados

BR-020 km 18, CEP: 73301-970 Planaltina/DF/Brasil

elaneborges@uefs.br; edson.sano@embrapa.br

\section{RESUMO}

Séries temporais têm possibilitado a identificação de mudanças no uso do solo e a discriminação de fitofisionomias. Este estudo objetivou utilizar séries temporais de índice de vegetação realçado (EVI) da plataforma Terra Modis, filtradas pelas técnicas de logística dupla e fração mínima de ruído (MNF) e classificadas pelo algoritmo spectral angle mapper (SAM) para mapear o uso e cobertura vegetal do Oeste da Bahia. Séries temporais representativas das classes: Campo sujo, Cerrado ralo, Cerrado típico, Cerrado denso, Floresta estacional semidecidual, Floresta estacional decidual, Vegetação secundária, Cultura agrícola e Pastagem cultivada foram utilizadas como membros de referência na aplicação do algoritmo SAM. A acurácia do mapeamento foi analisada por meio de imagens do satélite RapidEye e coeficiente de concordância Kappa. O filtro de logística dupla e a MNF reduziram significativamente os ruídos presentes nas imagens. A classificação discriminou as classes supracitadas (índice Kappa $=0,8$ ), todavia, baseado em dados anteriores disponíveis na literatura, houve subestimação da Pastagem cultivada e superestimação do Cerrado típico. Esse estudo permitiu demonstrar o potencial de 
séries temporais do sensor Modis para discriminar classes de uso e cobertura vegetal representativas do Oeste da Bahia.

Palavras-chave: Índice de Vegetação; Cerrado; Minimum Noise Fraction; Spectral Angle Mapper.

\begin{abstract}
Temporal series have enabled the identification of changes in land use and the discrimination of phytophysiognomies. This study aimed at using time series of enhanced vegetation index (EVI) of Terra Modis platform, filtered by the double logistics and minimum noise fraction (MNF) algorithms and classified by the spectral angle mapper (SAM) technique to map land use and land cover (LULC) classes from western Bahia. Representative time series of shrub Savanna, sparse Savanna, typical Savanna, dense Savanna, seasonal forest, dry forest, secondary vegetation, croplands and pasturelands were used as reference members in applying the SAM algorithm. The accuracy of mapping was analyzed by RapidEye satellite images and the Kappa's coefficient of agreement. The double logistics and the MNF filters reduced significantly the noise in the images. The classification discriminated all LULC classes (Kappa index $=0.8$ ), however, there were, according to data available in the literature, underestimation of pasturelands and overestimation of typical Savanna. This study demonstrated the potential of time series of Modis sensor to discriminate representative LULC classes of western Bahia.
\end{abstract}

Keywords: Vegetation Index; Savanna; Minimum Noise Fraction; Spectral Angle Mapper.

\title{
1. INTRODUÇÃO
}

A mesorregião do Extremo Oeste da Bahia, pertencente ao bioma Cerrado (mosaico de formações campestres, savânicas e florestais) (RIBEIRO e WALTER, 2008), é marcada pela expansão da fronteira agrícola, principalmente a partir da década de 1980 (MENKE et al., 2009; SANO et al., 2011; FLORES et al., 2012; SANTOS et al., 2012). Até o início da década de 1980, as áreas antropizadas dessa região se restringiam basicamente à pecuária extensiva e à agricultura familiar. Atualmente é uma das principais fronteiras agrícolas do Brasil em termos de produção de grãos, notadamente soja, milho e algodão, para exportação.

O Oeste da Bahia destaca-se de outras regiões do bioma Cerrado pelo fato da agricultura de sequeiro não ter substituído, de forma determinante, áreas degradadas de pastagens cultivadas. De modo geral, as pastagens cultivadas ocorrem na porção leste da área de estudo, em terrenos relativamente ondulados e com precipitação média anual inferior a $1.000 \mathrm{~mm}$ (por exemplo, município de Brejolândia, precipitação média anual $=942 \mathrm{~mm}$ ) $($ SEI, 1999). As culturas agrícolas ocupam predominantemente a porção oeste da área de estudo, onde os terrenos são planos (chapadões) e a precipitação média anual é superior a $1.000 \mathrm{~mm}$ (por exemplo, município de São Desidério, precipitação média anual = $1.182 \mathrm{~mm})($ SEI, 1999). 
Pelo fato dessas áreas agrícolas ocuparem terrenos predominantemente arenosos, há uma grande preocupação em termos de sustentabilidade ambiental, notadamente em termos de perda de solos por erosão fluvial e eólica e contaminação de lençol freático por agrotóxicos e fertilizantes. Há, portanto, necessidade de monitoramento contínuo da expansão agrícola nessa região. Tal monitoramento tem sido realizado principalmente com base na análise de imagens dos satélites Landsat, Alos e CBERS (uma cena por ano e por órbita/ponto) (MENKE et al., 2009; SANO et al., 2011).

$\mathrm{O}$ uso de imagens monotemporais com periodicidade quinzenal/mensal, como são os casos dos satélites mencionados no parágrafo anterior, nessa área de estudo, apresenta limitações por causa da sazonalidade climática marcante e da dinâmica espaço-temporal acentuada em termos de ocupação agrícola. Cenas adquiridas em dias distintos do ano dificultam a interpretação de imagens, pois a mesma classe de cobertura vegetal pode apresentar padrões espectrais distintos em função da sua acentuada sazonalidade climática. Além disso, essas imagens, livres de cobertura de nuvens, são normalmente passíveis de serem adquiridas somente no pico da estação seca (junho a agosto, SANO et al., 2007).

Uma alternativa a essa limitação é o monitoramento da cobertura vegetal natural e antrópica por meio de séries temporais como as obtidas pelo sensor Moderate Resolution Imaging Spectroradiometer (Modis) que se encontra a bordo das plataformas Terra e Aqua. Esse sensor adquire imagens da superfície da Terra a cada 1-2 dias (JUSTICE et al., 2002; SOARES et al., 2007), permitindo uma melhor análise da dinâmica do uso e da cobertura vegetal da área de estudo (ANDERSON et al., 2005; WARDLOW et al., 2007; ANJOS et al., 2013). O sensor Modis disponibiliza, gratuitamente na rede mundial de computadores, vários produtos denominados ready-to-use (prontos para uso), tais como reflectância, temperatura de superfície, produtividade primária líquida e índices de vegetação.

Índices de vegetação derivados de satélites têm sido utilizados em uma ampla variedade de aplicações, cujo intuito é o monitoramento e caracterização da cobertura vegetal (e.g., O’CONNOR et al., 2012; HILL, 2013; MA et al., 2013). Eles correspondem às medidas radiométricas adimensionais na faixa óptica do espectro eletromagnético e estão interrelacionados com diferentes parâmetros vegetacionais do dossel, tais como teor de clorofila, área foliar, biomassa verde, radiação fotossintética ativa e estrutura do dossel (JENSEN, 2007). Dezenas de índices têm sido propostos por diversos cientistas de diferentes países. Um dos mais populares é o índice de vegetação por diferença normalizada (NDVI) (TUCKER, 1979), o qual corresponde a uma relação entre a diferença de reflectância nas bandas espectrais do infravermelho próximo e do vermelho, dividido pela somatória das mesmas bandas. Outro índice bastante utilizado na literatura é o índice de vegetação realçado (EVI) (HUETE et al., 2002), o qual é mais resistente às variações dos efeitos do solo e da atmosfera. Esses dois índices fazem parte da lista dos produtos ready-to-use do sensor MODIS. 
Um grande número de estudos que analisa séries temporais de dados de satélites tem-se baseado na geração dessas séries a partir de amostras de pixels selecionadas ao longo de um determinado período de tempo (GALFORD et al., 2008; COUTO Jr. et al., 2013). Tais curvas podem ser utilizadas como membros de referência nos mapeamentos temáticos baseados em classificadores como o spectral angle mapper (SAM). O método SAM considera o ângulo de similaridade entre os membros de referência (KRUSE et al., 1993) e utiliza a premissa de que, quanto menor for o ângulo correspondente ao arco-cosseno (em radianos) entre os espectros, maior é a probabilidade deles pertencerem à mesma classe. $\mathrm{O}$ algoritmo SAM, embora desenvolvido para processar dados hiperespectrais, é passível de ser estendido para outras aplicações. Rowan e Mars (2003), por exemplo, utilizaram em estudo de exploração mineral para discriminar alvos a partir de dados do sensor ASTER na faixa do infravermelho termal. Carrino et al. (2011) aplicaram em dados provenientes de gamaespectroscopia para discriminação de áreas de espesso regolito. Vasconcelos et al. (2012) extraíram assinaturas geomorfométricas a partir de diferentes curvaturas de terrenos e realizaram a classificação geomorfológica por meio desse algoritmo. Cattani et al. (2013) avaliaram o referido algoritmo para classificar imagens de alta resolução espacial do satélite RapidEye. Em todos os trabalhos supracitados, os resultados obtidos, a partir do método SAM, foram considerados satisfatórios.

Uma das características inerentes às séries temporais é a presença de ruídos que são causados, por exemplo, no caso do sensor MODIS, por interferências da atmosfera (cobertura de nuvens) e/ou por ângulos de incidência do sensor relativamente elevados. Diversos métodos de suavização de séries temporais têm sido propostos, tais como a média móvel, a mediana, a análise harmônica, a transformada de Fourier, a transformada de Wavelet ou o filtro Savitzky-Golay (CHEN et al., 2004; CARVALHO Jr. et al., 2006; BRADLEY et al., 2007; LU et al., 2007; GALFORD et al., 2008; HIRD e McDERMID, 2009; COUTO Jr. et al., 2012). Cada filtro apresenta vantagens e desvantagens em relação às outras. Por exemplo, a média móvel é fácil de ser implementada e a sua execução é rápida, porém, os resultados da suavização deixam a desejar para dados com picos estreitos (CERQUEIRA et al., 2000). Ainda segundo esses últimos autores, a principal limitação da transformada de Fourier ocorre quando há variações bruscas na série temporal (por exemplo, quando uma área de Cerrado remanescente é convertida em cultura agrícola). Portanto, a escolha do filtro mais adequado para suavizar as séries temporais de interesse constitui-se em uma das etapas de pré-processamento que deve ser considerada em estudos que envolvem essas séries.

Outro aspecto importante é o fato de que as séries temporais geralmente apresentam grande quantidade de dados. Por isso, é recomendável o uso de técnicas que reduzem a dimensionalidade dos dados, sem perdas significativas de informações. Uma das abordagens mais comuns para reduzir a dimensionalidade dos dados é a técnica de componentes principais, a qual envolve rotação e translação linear de eixos, de forma a concentrar quantidades maiores de informações nos 
primeiros componentes ou fatores (INGEBRITSEN e LYON, 1985). Outra abordagem, derivada dos componentes principais, é o método de minimum noise fraction (MNF), proposto por Green et al. (1988) e modificado por Boardman e Kruse (1994). Além da redução da dimensionalidade dos dados, outra vantagem do MNF é a eliminação de informações com variâncias menores, o que minimiza ruídos sem degradar o sinal (GREEN et al., 1988; CARVALHO Jr. et al., 2002). No Brasil, ela tem sido aplicada na análise de séries temporais de sensores multiespectrais e com resolução espacial moderada (CARVALHO Jr. et al., 2012; COUTO Jr. et al., 2013). Além disso, o MNF leva em consideração os padrões espaciais repetitivos na série temporal para filtrar os dados. Desta forma, a aplicação desta técnica em séries temporais elimina a sobreposição das informações dos pixels em imagens de diferentes datas.

O grande desafio no uso de séries temporais para produzir mapas de uso e cobertura vegetal consiste em lidar com as mudanças que ocorrem em tempos distintos ao longo da série temporal, as quais estão relacionadas, por exemplo, com desmatamentos, queimadas, mudanças de usos e de práticas de manejo. Tal dinâmica implica em feições nas séries que não são inerentes ao comportamento dos alvos naturais, mas às intervenções antrópicas associadas às características dos alvos. Consequentemente, o uso das séries temporais tem sido enfatizado na identificação pontual de mudanças ao longo dessas séries que estariam associadas a interferências humanas (e.g., ANJOS et al., 2013).

Neste contexto, o objetivo desta pesquisa consistiu em utilizar séries temporais de EVI da plataforma Terra Modis, para mapear classes de uso e cobertura vegetal representativas do Oeste da Bahia. A hipótese a ser investigada é a de que as séries temporais funcionam a contento na área de estudo como membros de referência e, por conseguinte, podem ser utilizadas na classificação de alvos representativos dessa área.

\section{MATERIAIS E MÉTODOS}

A área de estudo desta pesquisa compreende a mesorregião do Extremo Oeste da Bahia, doravante denominada de Oeste da Bahia (Figura 1), é composta por vinte e quatro municípios e está localizada aproximadamente entre as coordenadas $10,05^{\circ}$ e $15,30^{\circ}$ de latitude sul e entre $43,25^{\circ}$ e $46,70^{\circ}$ de longitude oeste. Essa região é caracterizada ainda pelo clima tropical continental (Aw na classificação de Köppen) e pelos efeitos marcantes da sazonalidade climática. O Oeste da Bahia abrange uma área de aproximadamente $117 \mathrm{mil} \mathrm{km}^{2}$, situa-se na margem esquerda do rio São Francisco e tem, como afluentes principais, os rios Grande, Preto, Corrente e Carinhanha (NOU e COSTA, 1994).

Essa pesquisa foi baseada nos dados provenientes da plataforma Terra Modis. O processamento desses dados foi realizado nos aplicativos computacionais de processamento de imagens ENVI ${ }^{\mathrm{TM}}$ (versão 4.7) (software comercial) e de filtragem de séries temporais Timesat (versão 3.1.1) (domínio público, disponível para download na rede mundial de computadores). Os procedimentos metodológicos 
utilizados estão sintetizados na Figura 2 e são descritos a seguir. A base de dados desta pesquisa compreendeu uma série temporal de doze anos de EVI (2000 a 2011), com imagens compostas a cada 16 dias, resultando em 23 imagens por ano e em uma série temporal de 276 imagens, com resolução espacial de $250 \mathrm{~m}$. Estas cenas foram integradas em ordem cronológica. O EVI (HUETE et al., 2002) foi desenvolvido para realçar as respostas espectrais relacionadas com cobertura vegetal verde por meio da minimização dos efeitos de solos e da atmosfera. A equação é dada por:

$$
E V I=2,5 \frac{\rho_{I V P}-\rho_{V M}}{\rho_{I V P}+\left(6 \rho_{V M}-7,5 \rho_{A Z}\right)+1}
$$

Onde $\rho_{A Z}=$ reflectância na banda espectral do azul (MODIS banda 3); $\rho_{V M}=$ reflectância na banda espectral do vermelho (MODIS banda 1); e $\rho_{I V P}=$ reflectância na banda espectral do infravermelho próximo (MODIS banda 2).

Figura 1 - Localização da mesorregião do Extremo Oeste da Bahia, com destaque

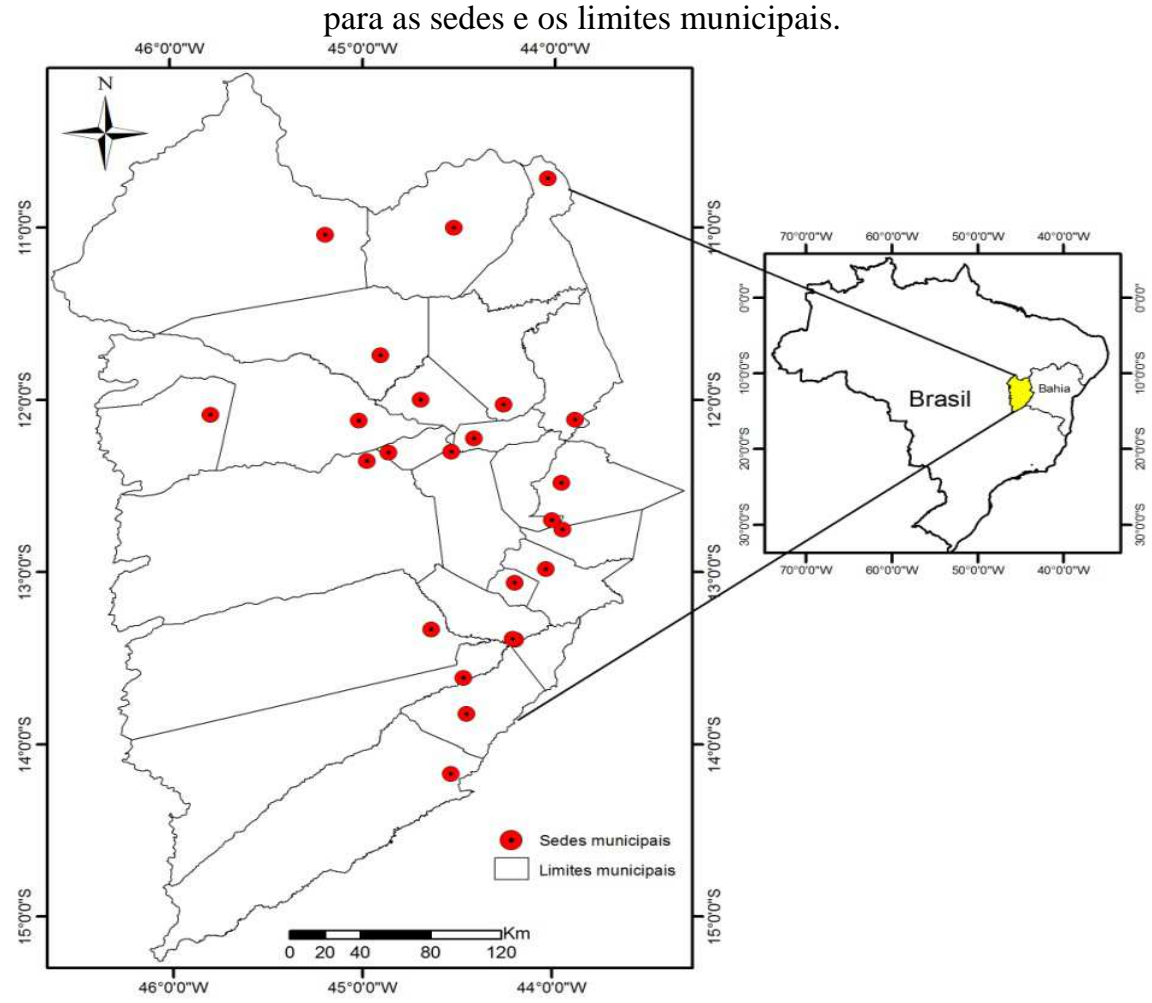

Bol. Ciênc. Geod., sec. Artigos, Curitiba, v. 20, nº 3, p.526-547, jul-set, 2014. 
Figura 2 - Fluxograma que sintetiza os procedimentos metodológicos utilizados na pesquisa.

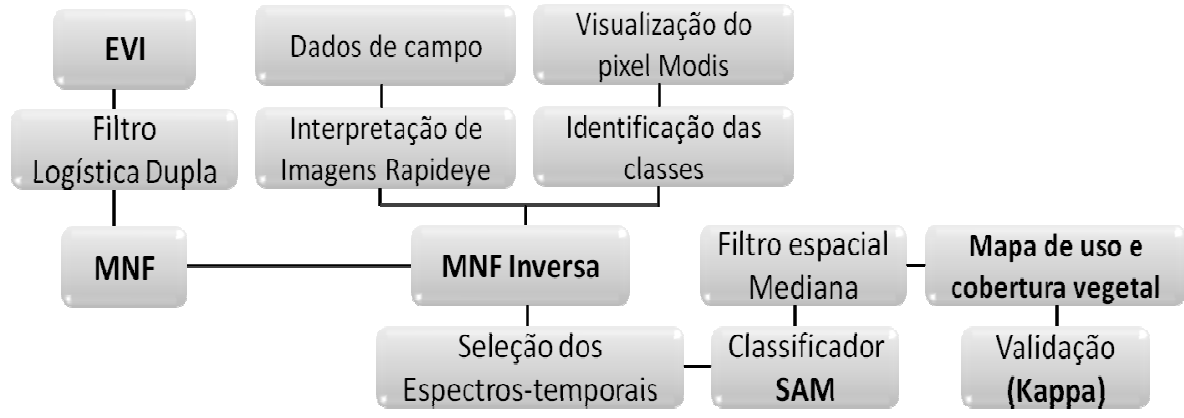

Os ruídos associados à série temporal da área de estudo foram suavizados por meio da aplicação do filtro logística dupla, implementado no Timesat. O Timesat foi selecionado por apresentar as seguintes vantagens (JONSSON e EKLUNDH, 2004; EKLUNDH e JONSSON, 2012): contém três opções de suavização ao invés de apenas uma; contém vários métodos de detecção de ruídos; e opção de processamentos individuais para diferentes classes de uso e cobertura de terras. Além disso, o Timesat não requer conhecimentos sólidos de linguagem de programação, frequentemente necessários para implementar os algoritmos desejados. Das três opções de suavizadores disponíveis no Timesat (logística dupla, Saviztky-Golay e gaussiano assimétrico), utilizou-se o filtro de logística dupla, o qual corresponde a uma função harmônica polinomial (Eq. 2).

$$
g\left(t ; x_{1}, \ldots, x_{4}\right)=\frac{1}{1+\exp \left(\frac{x_{1}-t}{x_{2}}\right)}-\frac{1}{1+\exp \left(\frac{x_{3}-t}{x_{4}}\right)}
$$

Onde $\mathrm{g}(\mathrm{t}, \mathrm{x})$ determinam a forma da base da função, sendo que o parâmetro $\mathrm{x}_{1}$ determina a posição do ponto de inflexão esquerdo, enquanto $\mathrm{x}_{2}$ fornece a taxa de variação. Similarmente, $x_{3}$ determina a posição do ponto de inflexão do lado direito enquanto $\mathrm{x}_{4}$ fornece a taxa de variação nesse ponto. Nesta função, os parâmetros utilizados são restritos, por isso garantem uma forma suave às séries temporais (JÖNSSON et al., 2004). A escolha da logística dupla foi em função do seu melhor desempenho estatístico, superior na suavização dos dados do Oeste da Bahia em relação aos outros dois filtros (BORGES, 2014). A Figura 3 mostra um exemplo de suavização da série temporal correspondente à classe Cerrado denso, uma das fitofisionomias do bioma Cerrado que é encontrada na região de estudo. 
Figura 3 - Exemplo de séries temporais de EVI original, EVI filtrado (logística dupla) e EVI filtrado mais MNF, representativas da classe Cerrado denso no município de Riachão das Neves.

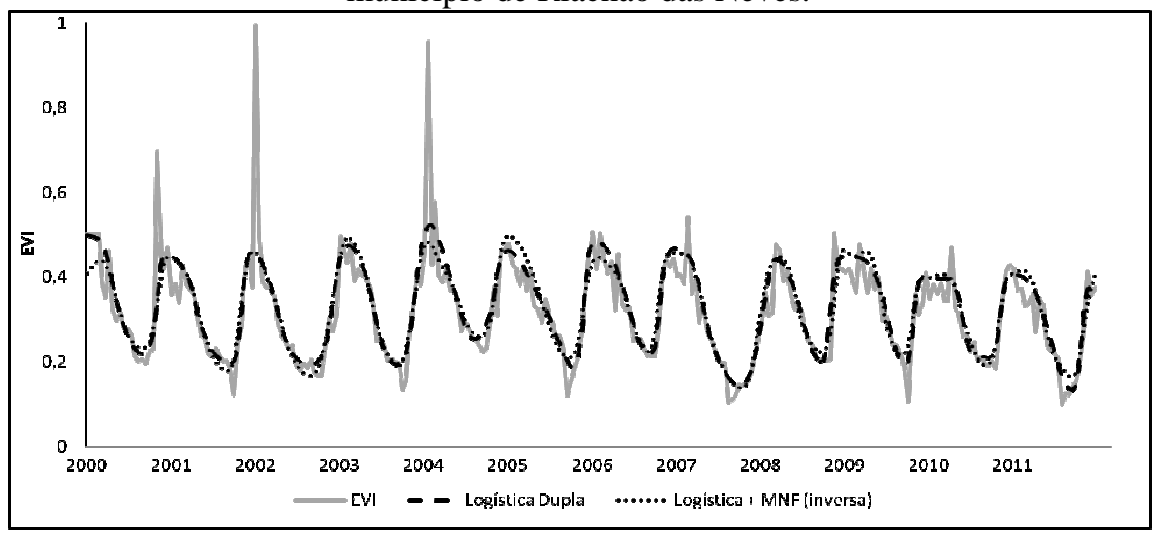

A série temporal de EVI filtrada foi submetida ainda à técnica de MNF. Conforme mencionado anteriormente, este procedimento reduz a dimensionalidade dos dados, isto é, minimiza a presença de ruídos, sem degradar o sinal. Após a $\mathrm{MNF}$, foi aplicada a MNF inversa, a qual re-escalona as informações concentradas nas primeiras frações selecionadas para o total de imagens utilizadas (BAPTISTA, 2012). Conforme a interpretação dos dados estatísticos de autovalores, utilizaram-se as 50 primeiras frações resultantes da MNF para gerar a transformação da $\mathrm{MNF}$ inversa.

Esse conjunto de frações foi classificado com base no algoritmo SAM e em membros de referência (séries temporais representativas das classes de uso e cobertura de terras consideradas). O SAM utiliza os ângulos espectrais formados entre um espectro de referência e um espectro teste (pixel a ser classificado) (SOUTH et al., 2004). A equação do SAM é expressa por (KRUSE et al., 1993):

$$
\alpha=\cos ^{-1} \frac{\sum_{i=1}^{i=b} R E_{i}}{\sqrt{\sum_{i=1}^{i=b}\left(E_{i}\right)^{2} \sum_{i=1}^{i=b}}\left(R_{i}\right)^{2}}
$$

Onde $\alpha=$ função do arco-cosseno; quanto menor for $\alpha$, maior será a similaridade entre as curvas; $\mathrm{b}=$ número de bandas; $\mathrm{R}=$ espectro de referência; e $\mathrm{E}$ $=$ espectro teste.

Os membros de referência foram obtidos com base nos trabalhos de campo realizados no mês de maio de 2012 para inspeção visual das classes de uso e cobertura vegetal representativas da área de estudo. Foram visitados um total de 86 pontos nos municípios de Barreiras e Luís Eduardo Magalhães. Em cada área, foi 
identificada a denominação correta da classe e obtidas as coordenadas com aparelho receptor de GPS (Global Positioning Satellite) e fotografias digitais panorâmicas. As seguintes classes temáticas representativas da área de estudo foram identificadas e selecionadas para análises posteriores: Campo sujo, Cerrado ralo, Cerrado típico, Cerrado denso, Floresta estacional semidecidual, Floresta estacional decidual (popularmente conhecida como Mata seca), Vegetação secundária, Cultura agrícola e Pastagem cultivada (Figura 4).

Como a classe Cultura agrícola apresenta diferentes culturas e ciclos vegetativos, foram selecionadas três curvas como sendo representativas desta classe: Cultura de sequeiro; Cultura de sequeiro com safrinha, cuja série é caracterizada pela curva bimodal; e a Agricultura familiar, dominante na região sudeste da área de estudo e com maior heterogeneidade de culturas em relação à borda ocidental da área de estudo, bem como apresenta poligonais de cultivos menores. A extração de médias aritméticas dos valores de EVI presentes nas séries temporais de uma determinada classe poderia camuflar algumas especificidades, gerando valores que não caracterizam corretamente a classe. Para as demais classes, selecionou-se apenas uma curva temporal.

Figura 4 - Fotografias registradas em trabalho de campo representativas das fitofisionomias e usos existentes na área de estudo: a) Campo sujo; b) Cerrado ralo;

c) Cerrado típico, d) Cerrado denso; e) Floresta estacional decidual; f) Floresta estacional semidecidual; g) Vegetação secundária; h) Cultura agrícola; i) Pastagem cultivada.
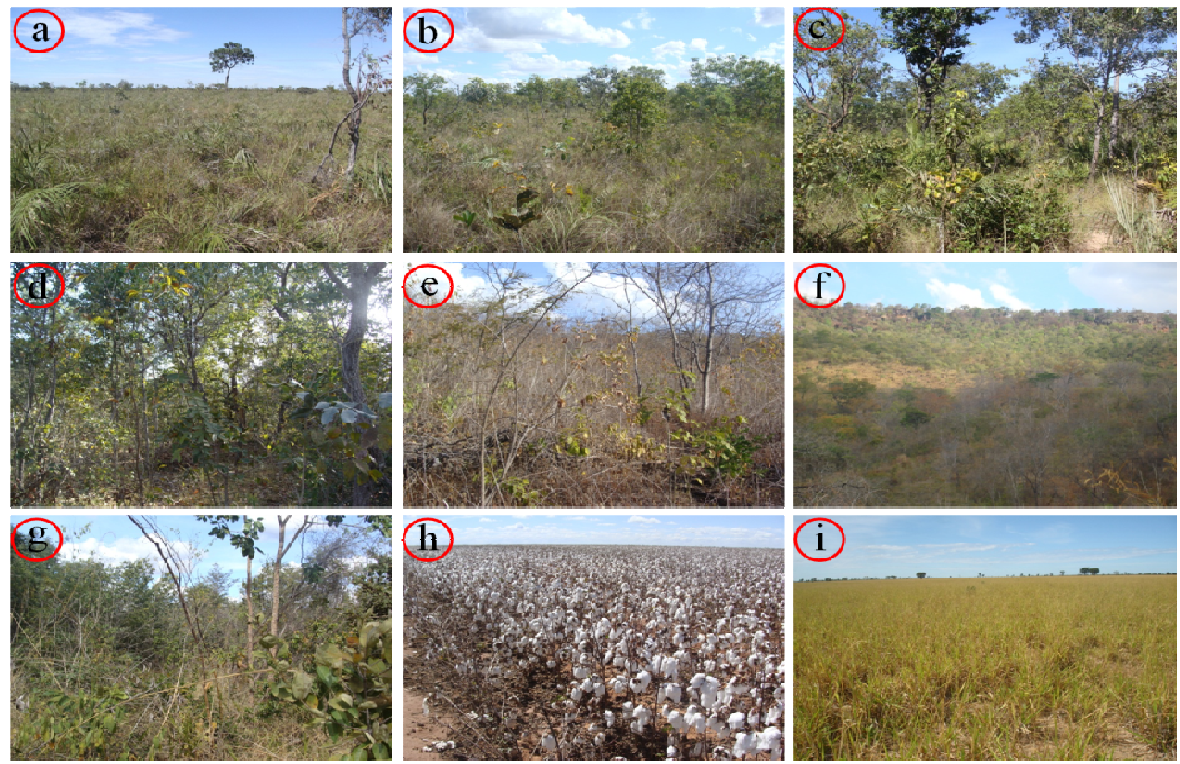

Bol. Ciênc. Geod., sec. Artigos, Curitiba, v. 20, nº 3, p.526-547, jul-set, 2014. 
Posteriormente, criou-se uma biblioteca de valores temporais de EVI (membros de referência) das classes representativas da área de estudo (Figura 5). Para isso, foi utilizada a plataforma instantânea de visualização de EVI2, desenvolvida por Freitas et al. (2011) e disponibilizada na página eletrônica do Instituto Nacional de Pesquisas Espaciais (INPE). Nessa plataforma, é possível visualizar os limites dos pixels individuais do MODIS sobrepostos nas imagens de satélites disponíveis no programa Google Earth ${ }^{\mathrm{TM}}$. Essa plataforma foi utilizada nesse estudo para certificar de que os membros de referência correspondiam a pixels "puros", isto é, sem mistura de diferentes classes de uso e cobertura vegetal.

Figura 5 - Valores temporais de EVI (membros de referência) (2000-2011) representativos das classes identificadas pelo algoritmo SAM.

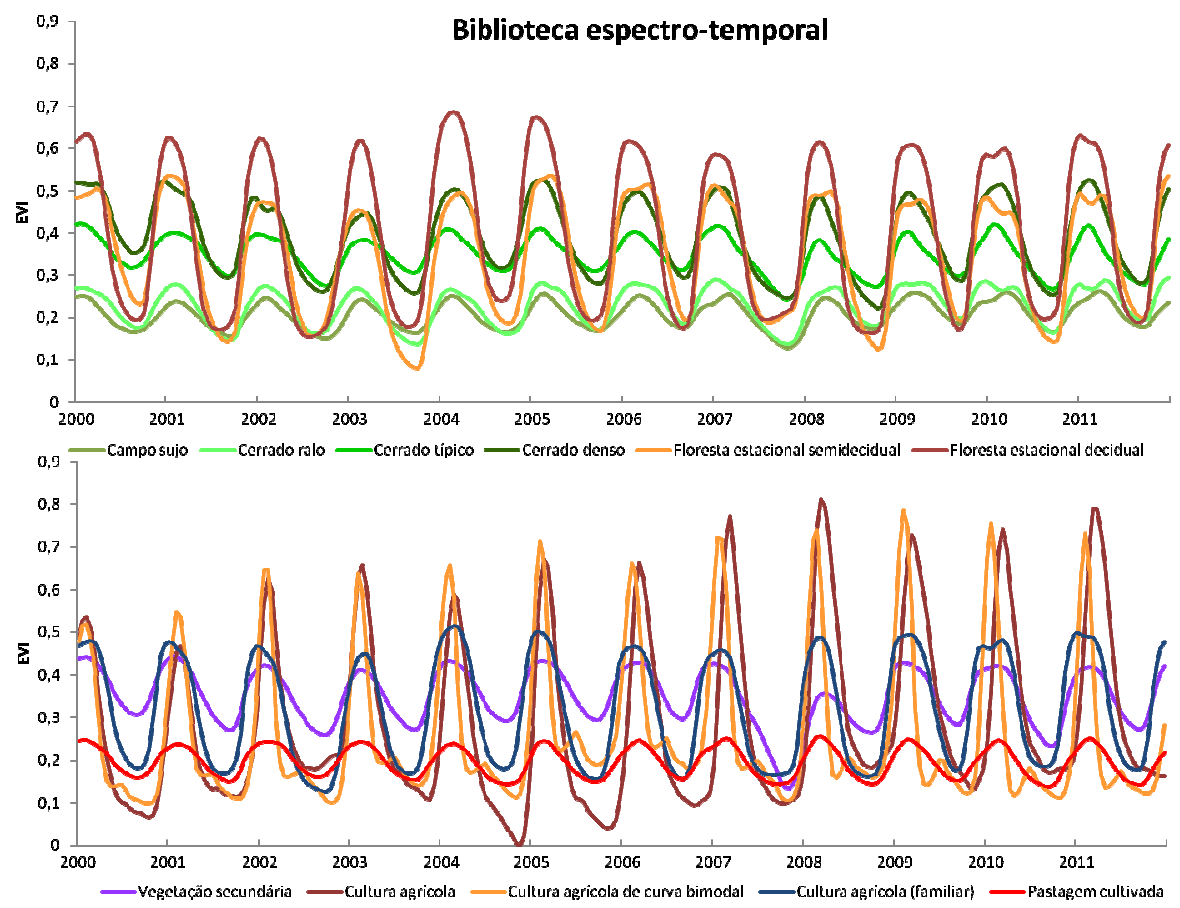

Essas curvas foram utilizadas para classificar as imagens do MODIS EVI, processadas pela técnica de MNF inversa, por meio do algoritmo SAM. Esse algoritmo gera produtos denominados rules images que mostram valores de ângulos dos pixels, possibilitando identificar o ângulo mais favorável para cada membro de referência. Desta maneira, foram especificados diferentes ângulos para cada classe. Todavia, a grande maioria dos pixels não foi classificada, sendo apenas alguns 
pixels mapeados como pertencentes a cada classe. Optou-se por não especificar o valor do ângulo para cada classe, pois a referida especificação estava gerando número excessivo de pixels não-classificados. Isto ocorre por causa da intensa dinâmica de ocupação humana na região e da resolução espacial do Modis, fazendo com que muitos pixels de EVI sejam, por exemplo, uma combinação de duas ou mais classes de coberturas de terras existentes na área de estudo.

Após a classificação da série temporal, por meio do algoritmo SAM, aplicouse um filtro espacial de mediana com janela de $3 \times 3$ pixels para a remoção de pixels isolados e homogeneização do resultado da classificação. A última etapa deste trabalho consistiu na validação da classificação para verificação da acurácia do mapeamento. Foram selecionados, aleatoriamente, 100 pontos no mapa de uso e cobertura vegetal e validados com base nas imagens dos satélites RapidEye. Esse satélite opera com uma constelação de cinco satélites idênticos que estão posicionados no espaço em uma mesma órbita solssíncrona (TYC et al., 2005). O RapidEye opera com cinco bandas (faixas espectrais do azul, verde, vermelho, rededge e infravermelho próximo), resolução espacial nominal de 6,5 metros, 12 bits de resolução radiométrica e tempo de revisita diária (fora do nadir) ou de 5,5 dias (a nadir). Cada cena ortorretificada e reamostrada para um tamanho de pixel de 5 metros, corresponde a uma área no terreno de 25 x $25 \mathrm{~km}$. Foram consideradas 260 imagens RapidEye, referentes ao ano de 2011.

A validação do mapeamento temático foi baseada no cálculo do índice de concordância Kappa (LANDIS e KOCH, 1977). Essa medida de concordância varia de $<0$ (nenhuma concordância) a 1 (concordância total). Esses dois autores sugeriram a seguinte interpretação do índice Kappa: $<0=$ sem concordância; 0 $0,19=$ concordância pobre; $0,20-0,39=$ concordância razoável; $0,40-0,59=$ concordância moderada; $0,60-0,79=$ concordância substancial; e $0,80-1=$ concordância quase perfeita.

\section{RESULTADOS E DISCUSSÃO}

Os resultados indicaram uma redução significativa dos ruídos com as aplicações do filtro de logística dupla e da técnica MNF. A título de ilustração, na Figura 6, são mostradas a composição colorida das frações MNF 3, 4 e 5, a primeira e a $276^{a}$ fração MNF. As informações concentram-se nas primeiras frações, em detrimento das últimas, cujos dados são mais ruidosos. Na Figura 6a, podem-se observar três ambientes bem diferentes, as áreas correspondentes ao uso agropecuário (a oeste, representada por cores variadas, tais como vermelho, amarelo e ciano), áreas de predomínio da vegetação savânica (porção central, onde predominam as cores em tons de verde) e ambientes com predominância da vegetação estacional decidual e semidecidual (a leste, representadas pelas cores rosa e amarelo). A Figura $6 \mathrm{~b}$ consiste na primeira fração MNF, a qual concentra a maior quantidade de informação. A parte mais escura, localizada predominante na porção oeste, corresponde basicamente a áreas de culturas agrícolas e fitofisionomias predominantemente savânicas. A parte mais clara, localizada preferencialmente na 
leste da área de estudo, correspondem a fitofisionomias predominantemente florestais. A Figura $6 c$ corresponde à última fração MNF, ou seja, à imagem mais ruidosa.

A classe Campo sujo correspondeu a 1,8\% da área de estudo e apresentou valores temporais de EVI muito similares aos da pastagem cultivada, por isso houve confusão destas classes, visto que os pixels classificados como Campo sujo apresentaram elevado desvio padrão (Figura 7). Nesta fitofisionomia, predomina a vegetação de gramíneas, a qual responde rapidamente à precipitação na estação chuvosa, assim como à falta dela durante a seca. Este intenso padrão sazonal dificulta a separação entre mudanças devido às causas antrópicas (queimadas) e mudanças fenológicas da cobertura vegetal quando se utilizam imagens de uma única data (ANDERSON et al., 2005), por isso a série temporal de EVI oferece grande potencial na discriminação dessa fitofisionomia, pois considera o seu comportamento sazonal durante toda a serie temporal.

Figura 6 - Composição colorida das frações 3, 4 e 5 derivadas do processamento de imagens por MNF em R, G e B (a), primeira fração MNF (b) e $276^{a}$ fração MNF (c).

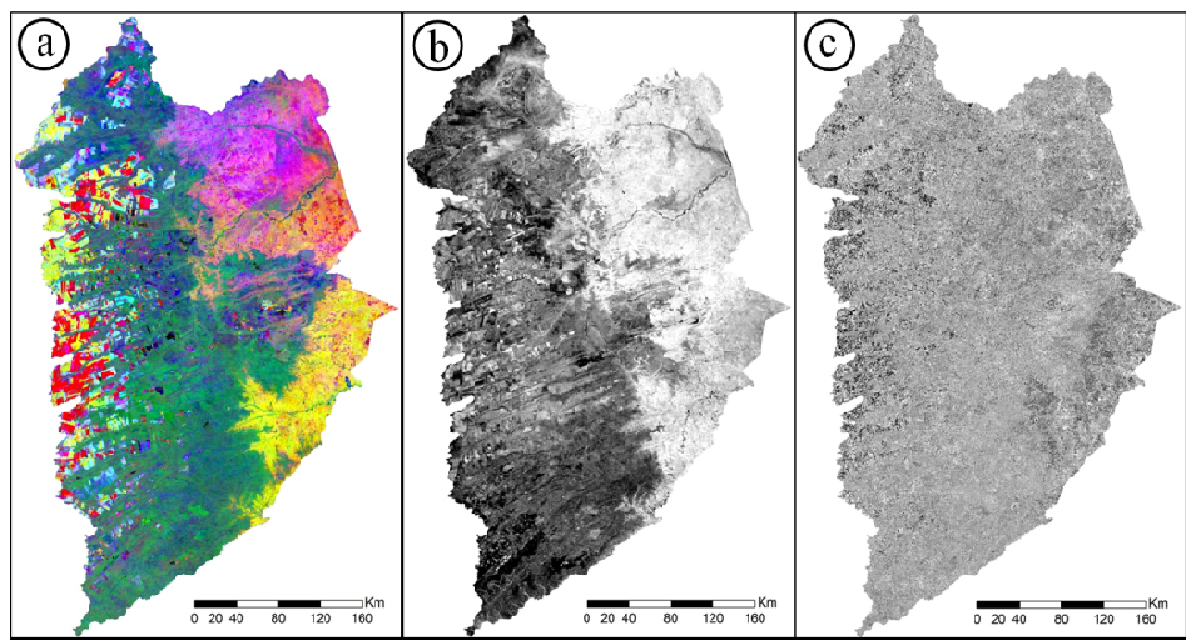

O Cerrado ralo, em relação ao Cerrado típico, distingue-se quanto à densidade da vegetação, portanto, as curvas ora se assemelham, ora se diferenciam em alguns aspectos, tais como valores de EVI mais elevados. Áreas de Cerrado ralo podem ter sido, no passado não muito remoto, Cerrado típico, haja vista que o que distingue essas duas classes são a densidade e a altura das árvores e arbustos (RIBEIRO e WALTER, 2008). Identificaram-se 5,1\% como pertencente à classe de Cerrado ralo na área de estudo e o desvio padrão dos valores temporais de EVI foi mediano, comparado aos das demais classes. 
O Cerrado típico é a fitofisionomia predominante, distribuída na porção central da área de estudo e corresponde a 42,8\%. Essa fitofisionomia é intermediária entre o Cerrado denso e o Cerrado ralo (RIBEIRO e WALTER, 2008), corresponde à maior representação de Cerrado ainda existente no Oeste da Bahia, mas é, proporcionalmente, a vegetação mais suprimida, tendo em vista as características das áreas onde ocorrem - topografia plana e solos bem desenvolvidos - as quais proporcionam o avanço da agricultura mecanizada em larga escala. A série temporal de EVI para esta classe apresentou baixo desvio padrão.

A classe Cerrado denso constitui-se em manchas mais esparsas e localiza-se principalmente nas proximidades das bordas dos chapadões e representa 9,6\% da área de estudo. Esta fitofisionomia é caracterizada por arbustos e árvores, porém, com densidade maior do que a encontrada no Cerrado típico. Os pixels classificados como pertencentes a esta classe apresentaram desvio padrão mediano quando comparado aos das demais classes (Figura 7).

Figura 7 - Desvio padrão dos valores temporais de EVI dos pixels identificados em cada classe de uso e cobertura vegetal considerada nesse estudo.
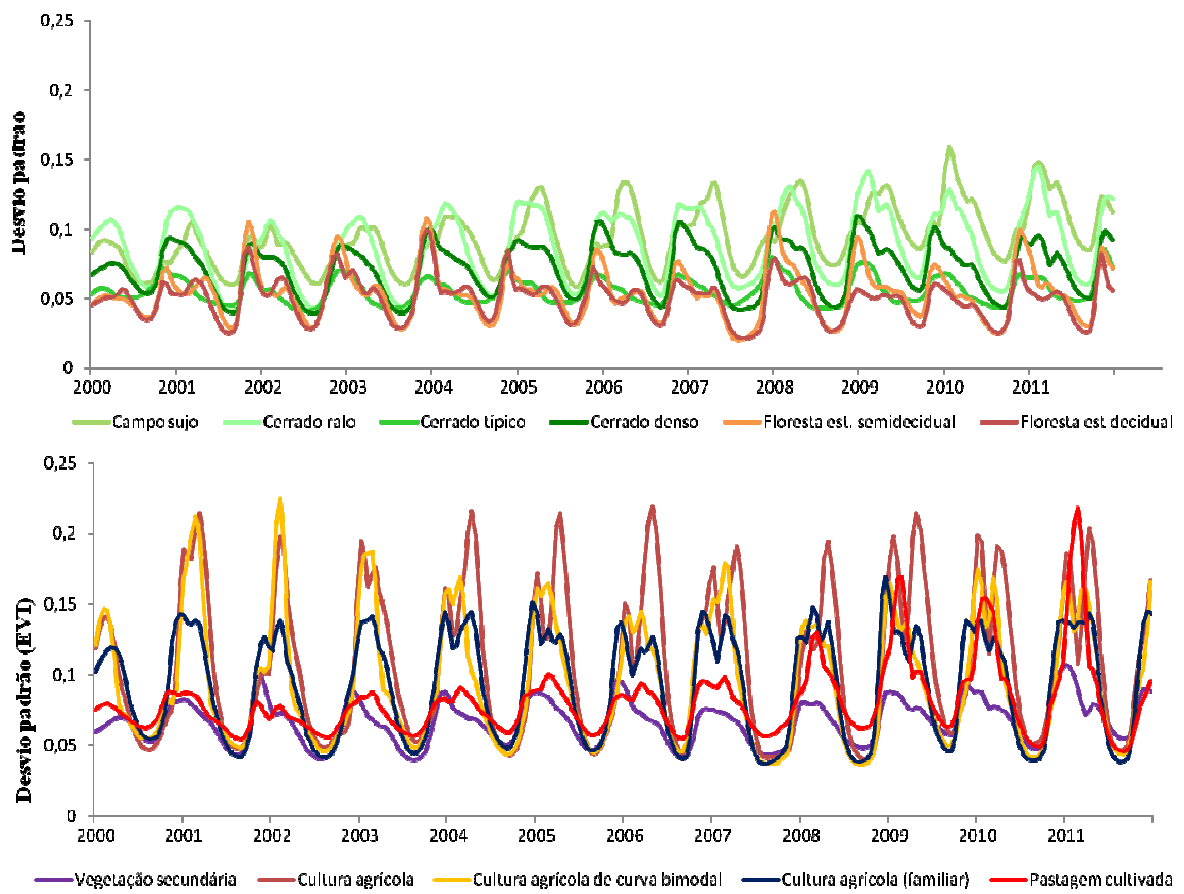

Bol. Ciênc. Geod., sec. Artigos, Curitiba, v. 20, nº 3, p.526-547, jul-set, 2014. 
A Floresta estacional decidual e a Floresta estacional semidecidual constituemse em alvos bastante marcados pela sazonalidade e ocupam principalmente a unidade geomorfológica do pediplano sertanejo (BRASIL, 2003). A área abrangida por estas fitofisionomias é equivalente a $7,8 \%$ para Floresta estacional decidual e $10,6 \%$ para Floresta estacional semidecidual. Os pixels pertencentes a estas classes apresentaram baixo desvio padrão, visto que suas curvas temporais são bem características na série de EVI.

A Vegetação secundária corresponde às áreas cuja vegetação sofreu ação antrópica, como desmatamento ou queimada, e depois entrou em processo de regeneração, sendo, na área de estudo, mapeados $2 \%$ como pertencente a esta classe. A maior parte das áreas de vegetação nativa que foi desmatada ou queimada foi convertida posteriormente em áreas agrícolas ou pastagens, não sendo possível a regeneração da vegetação. O desvio padrão dos pixels classificados como pertencentes a esta classe foi baixo.

A classe Cultura agrícola, caracterizada principalmente pela agricultura de sequeiro, se concentra predominantemente na parte ocidental da área de estudo, mas também a nordeste e sudeste, porém, nestas regiões, predomina a agricultura familiar, que é mais diversificada em termos de tipos de culturas. A curva temporal extraída da Agricultura familiar é caracterizada por menor amplitude e maior comprimento em relação à curva temporal da Agricultura de sequeiro que é predominante na borda oeste da área de estudo (Figura 6), pois nestas áreas têm-se ciclos de culturas mais curtos, devido às modernas técnicas utilizadas. Esta classe avançou para as áreas de baixas declividades e solos profundos, principalmente latossolos, denominadas de chapadões. As curvas temporais representativas da classe Cultura agrícola foram as que apresentaram maior desvio padrão, isto porque se tem, ao longo da série, mudanças em relação aos tipos de cultivos e, consequentemente, na fenologia, no tipo de dossel, e na densidade de indivíduos, dentre outras alterações.

$\mathrm{Na}$ região de estudo, tradicionalmente predominava o uso da pecuária extensiva e da agricultura familiar, todavia, estas áreas, ao longo do tempo, tornaram-se mais restritas, preponderando a Pastagem cultivada. Esta classe corresponde a $2,8 \%$ da área de estudo, mas algumas áreas antropizadas em torno das áreas de agricultura de sequeiro foram identificadas como Pastagem cultivada e algumas áreas de Pastagens cultivadas foram classificadas como Culturas agrícolas.

As classes antrópicas apresentaram maior complexidade de identificação porque o mesmo tipo de uso pode ocorrer em distintos períodos, não sendo passível de se caracterizar todas as curvas espectro-temporais como representativas da mesma classe. Classes antrópicas implicam em diversos processos/eventos que podem acontecer de forma diferenciada no tempo e no espaço.

Alguns erros de classificação ocorreram por causa da resolução espacial moderada do Modis. Determinadas manchas, menores do que o tamanho nominal do pixel, não são possíveis de serem identificadas ou caracterizadas como tais. Piromal et al. (2008), por exemplo, encontraram erros grosseiros de classificação quando 
utilizaram o produto MOD14 para identificar queimadas na Amazônia, não sendo possível mapear áreas menores que 100 hectares, devido à relativamente baixa resolução espacial das imagens utilizadas.

Por meio do desvio padrão, pôde-se observar como as curvas temporais de EVI dos pixels mapeados para cada fitofisionomia e tipos de usos variaram temporalmente (Figura 7). Conforme esperado, as classes antrópicas apresentaram maior desvio padrão, o que indica maior variabilidade dos pixels em função da alternância de culturas agrícolas ou mudanças de uso. As classes naturais, por sua vez, apresentaram menor desvio padrão, pois os pixels pertencentes a esta classe mantém comportamento similar entre si durante o período analisado.

A seleção de membros de referência de forma manual apresentou melhor resultado do que a realizada de forma semiautomática (por exemplo, Pixel Purity Index e $\mathrm{N}$-Dimensional). Resultado semelhante foi encontrado por Rosso et al. (2005).

Na Figura 8, é apresentado o mapa de uso e cobertura vegetal do Oeste da Bahia. Como se trata de curvas temporais, e não de espectro de reflectância, o método de classificação SAM não foi aplicado de forma rigorosa, isto é, não se arbitrou um ângulo de similaridade, sendo todos os pixels da imagem incluídos na classe que detém o conjunto de pixels com curvas temporais mais semelhantes. A não especificação do ângulo para aplicação do método SAM pode provocar generalizações de classes, pois a utilização deste algoritmo permitiria identificar apenas as áreas que possuem pixels com espectros similares, conforme o ângulo estabelecido para cada membro de referência.

Os comportamentos sazonais, discriminados pelos valores temporais de EVI das diferentes fitofisionomias e das classes de uso, foram imprescindíveis na identificação e caracterização das mesmas. A despeito disto, pode-se afirmar que o efeito da sazonalidade poderia ser identificado de forma ainda mais marcante na área de estudo, porém, o produto MOD13Q1 utiliza a imagem de EVI mais elevado para fazer a composição a cada 16 dias (LEEUWEN et al. 1999).

Algumas áreas recentemente antropizadas foram classificadas conforme a classe de vegetação pre-existente, pois foi considerado o padrão temporal predominante nestes pixels. Resultados encontrados por Sano et al. (2011), por meio de classificação de imagens TM/Landsat, demonstram que, em 2005, as áreas de Culturas agrícolas corresponderam a 1,8 milhões de hectares no Oeste da Bahia. Tais resultados estão condizentes com esta pesquisa que encontrou pouco mais de 2 milhões de hectares relativos a essa classe para 2011. Sano et al. (2011) também contabilizaram um total de 1,3 milhões de hectares de Pastagens cultivadas em 2005. Esse valor é bem maior que os 332 mil hectares obtidos com base nas séries temporais do Modis EVI. Acredita-se que essa subestimação pode ter sido causada pela confusão com Culturas agrícolas, principalmente do sudeste da área de estudo. Sano et al. (2009) também observaram confusão semelhante ao realizar mapeamento de uso e cobertura de terras no Distrito Federal. 
Figura 8 - Mapa de uso e cobertura vegetal do Oeste da Bahia gerado a partir da classificação SAM.

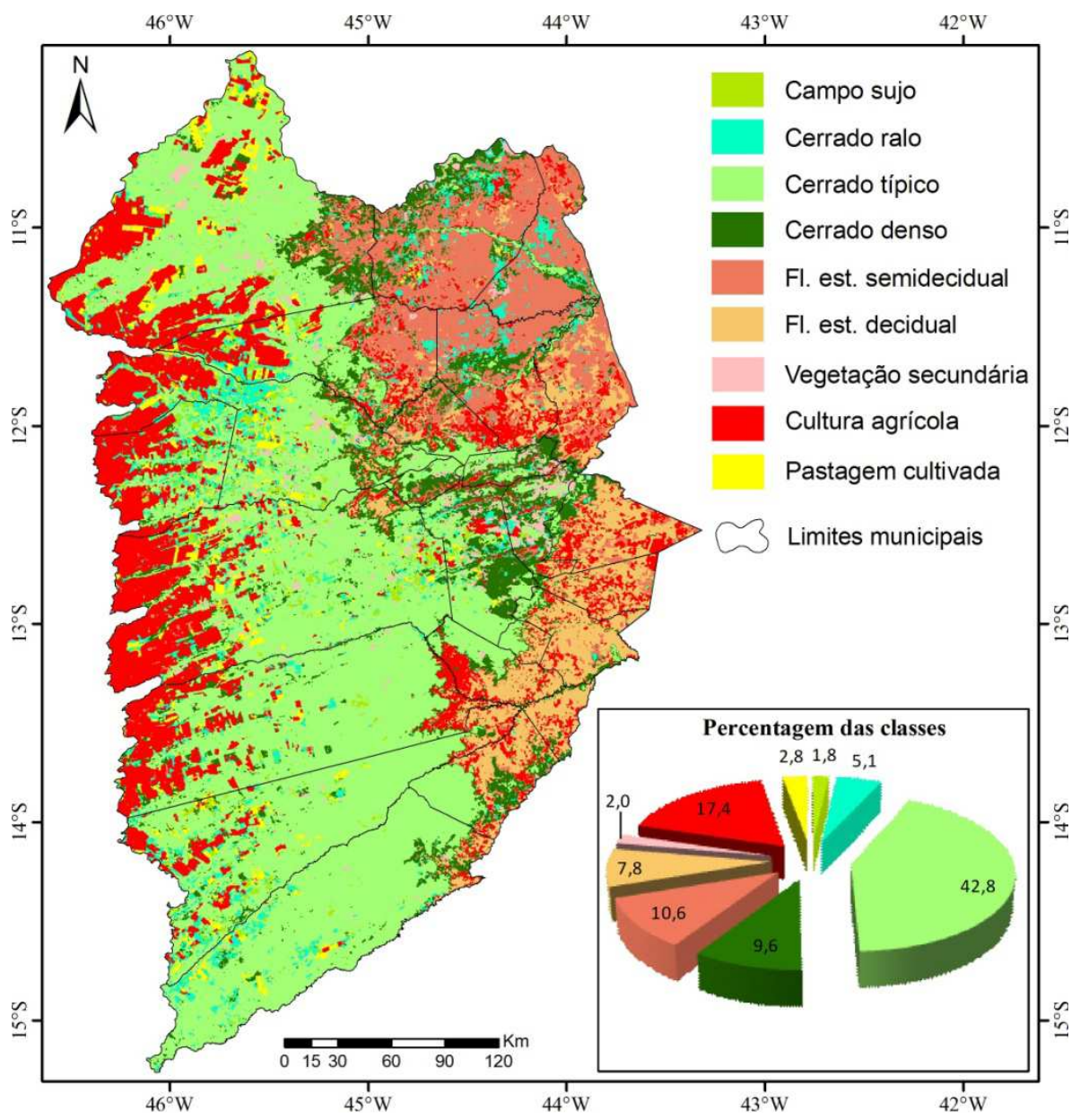

Do total de 100 pontos selecionados aleatoriamente no mapa de uso e cobertura vegetal para validação do mapeamento, 10 foram desconsiderados por corresponderem a pixels mistos nas imagens do satélite RapidEye. A Tabela 1 mostra a matriz de confusão. De um total de 90 pontos, 76 foram classificados corretamente, resultando em uma exatidão global de $84 \%$. O coeficiente de concordância Kappa encontrado foi de 0,8, considerado "muito bom", segundo a classificação de qualidade deste índice (LANDIS e KOCH, 1977). 
Tabela 1 - Matriz de confusão do mapeamento de uso e cobertura vegetal da área de estudo.

\begin{tabular}{|c|c|c|c|c|c|c|c|c|c|c|c|c|}
\hline \multirow{13}{*}{ 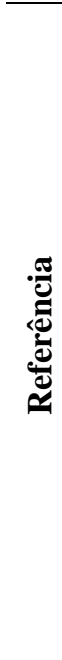 } & & \multicolumn{9}{|c|}{ Classificação } & \multirow[b]{2}{*}{ Total } & \multirow[b]{2}{*}{$\% \mathrm{EC}$} \\
\hline & & CS & CR & CT & CD & FS & FD & VS & CA & PC & & \\
\hline & CS & 1 & & & & & & & & & 1 & 0 \\
\hline & CR & & 2 & 2 & & & & & 1 & & 5 & 33 \\
\hline & $\mathbf{C T}$ & & & 32 & 1 & & & & & & 33 & 1,7 \\
\hline & CD & & & & 7 & & & & & & 7 & 0 \\
\hline & FS & & & & & 8 & & & & & 8 & 0 \\
\hline & FD & & & & & & 6 & & & & 6 & 0 \\
\hline & VS & & 2 & & & & & 2 & & & 4 & 27,5 \\
\hline & $\mathbf{C A}$ & 1 & & 1 & 2 & 1 & & & 15 & 1 & 21 & 15,7 \\
\hline & PC & 1 & 1 & & & & & & & 3 & 5 & 22 \\
\hline & Total & 3 & 5 & 35 & 10 & 9 & 6 & 2 & 16 & 4 & 90 & 100 \\
\hline & $\% \mathrm{EO}$ & 32,1 & 28,9 & 4,1 & 14,5 & 5,4 & 0 & 0 & 3 & 12 & 100 & \\
\hline
\end{tabular}

$\mathrm{CS}=$ Campo sujo; $\mathrm{CR}=$ Cerrado ralo; $\mathrm{CT}=$ Cerrado típico; $\mathrm{CD}=$ Cerrado denso; $\mathrm{FS}=$ Floresta estacional semidecidual; $\mathrm{FD}=$ Floresta estacional decidual; $\mathrm{VS}=$ Vegetação secundária; $\mathrm{CA}=$ Cultura agrícola; $\mathrm{PC}=$ Pastagem cultivada; $\mathrm{EC}=$ Erro de comissão $; \mathrm{EO}=$ Erro de omissão.

Ressalta-se que o mapeamento de uso e cobertura vegetal, realizado a partir de séries temporais de EVI do sensor Modis, utilizou imagens cuja resolução espacial foi $250 \mathrm{~m}$, portanto, os resultados devem ser analisados de acordo com a escala do mapa ( 1:1.000.000). As confusões de classes ocorreram em relação aos tipos de uso ou fitofisionomias, mas os dados do mapeamento mostraram-se coerentes quanto ao porte da vegetação (campestre, savânica e florestal) e à identificação de classes antrópicas. As classes de Campo sujo e Cerrado ralo foram as que apresentaram erros de omissão mais elevados, $32,1 \%$ e $28,1 \%$, respectivamente, sendo a classe de Cerrado ralo subestimada. As classes de Cerrado ralo, Vegetação secundária e Pastagens cultivadas apresentaram erros de comissão maiores: 33\%, $27,5 \%$ e $22 \%$, respectivamente.

\section{CONCLUSÕES}

Esse estudo permitiu demonstrar o potencial de séries temporais do sensor Modis para discriminar classes de uso e cobertura vegetal representativas do Oeste da Bahia, podendo ser aplicado em outras áreas. Este método demonstrou certa limitação para áreas naturais que foram antropizadas durante a série temporal considerada e também para áreas antropizadas que sofreram mudanças de uso (por exemplo, áreas de pastagens convertidas para culturas agrícolas).

Para a série temporal do Oeste da Bahia, recomenda-se o uso de um filtro temporal seguido da técnica MNF para suavização de ruídos.

Bol. Ciênc. Geod., sec. Artigos, Curitiba, v. 20, nº 3, p.526-547, jul-set, 2014. 


\section{AGRADECIMENTOS}

Os autores agradecem à Universidade Federal do Oeste da Bahia (UFOB) e à Universidade Estadual de Feira de Santana (UEFS), instituições de vínculo da primeira autora durante a pesquisa, pela viabilização desse estudo. Os autores são gratos ainda à Embrapa Cerrados, pelo apoio logístico e ao seu servidor Heleno Bezerra pelo apoio ao trabalho de campo. Ao Conselho Nacional de Desenvolvimento Científico e Tecnológico (CNPq), pelo fornecimento de bolsa de pesquisa ao segundo autor. As imagens RapidEye utilizadas nesse estudo foram adquiridas pelo Ministério do Meio Ambiente e, por meio de assinatura de termo de acesso a essas imagens, disponibilizadas para a realização dessa pesquisa.

\section{REFERÊNCIAS BIBLIOGRÁFICAS}

ANDERSON, L. O.; ARAGÃO, L. E. O. C.; LIMA, A.; SHIMABUKURO, Y. E. Detecção de cicatrizes de áreas queimadas baseada no modelo linear de mistura espectral e imagens índice de vegetação utilizando dados multitemporais do sensor MODIS/TERRA no estado do Mato Grosso, Amazônia brasileira. Acta Amazonica, v. 35(4), p. 445-456, 2005.

ANJOS, V. S.; SANO, E. E.; BEZERRA, H. S.; ROSA, R. Caracterização espectrotemporal de pastagens do Triângulo Mineiro utilizando dados MODIS EVI2 (2000-2010). Sociedade \& Natureza, v. 25(1), p. 205-215, 2013.

BAPTISTA, G. M. M. Processamento de dados hiperespectrais. IN: Introdução ao Processamento de Imagens de Sensoriamento Remoto. Org.: Paulo Roberto Meneses; Tati de Almeida. p. 221-238, Brasília, 2012. On-line (UnB/CNPq).

BORGES, E. F. Discriminação e caracterização fenológica de classes de cobertura vegetal natural e antrópica do Oeste da Bahia a partir de séries temporais do sensor Modis. 2014. 138 f. Tese (Doutorado em Geociências Aplicadas). Universidade de Brasília. Brasília, 2014.

BRADLEY, B. A.; JACOB, R. W.; HERMANCE, J. F.; MUSTARD, J. F. A curve fitting procedure to derive inter-annual phenologies from time series of noisy satellite NDVI data. Remote Sensing of Environment, v. 106(2), p. 137-145, 2007.

BRASIL. Companhia de Pesquisa de Recursos Minerais. Geologia e Recursos Minerais do Estado da Bahia: Sistema de Informações Geográficas - SIG e Mapas. CPRM, 2003. CD-ROM.

CARRINO, T. A.; SIlVA, A. M.; BOTElho, N. F.; SilVA, A. A. C. Discriminação de áreas de espesso regolito do leste do estado do Amazonas usando estatística multivariada, algoritmo hiperespectral e modelagem de dados espaciais. Revista Brasileira de Geofísica, v. 29(1), p. 155-172, 2011.

CARVALHO Jr., O. A.; CARVALHO, A. P. F.; MENESES, P. R.; GUIMARÃES, R. F. Classificação e eliminação dos ruídos em imagens hiperespectrais pela análise seqüencial da transformação por fração de ruído mínima. Revista Brasileira de Geofísica, v. 20(1), p. 31-41, 2002. 
CARVALHO Jr., O. A.; GUIMARÃES, R. F.; MARTINS, E. S.; CARVALHO, A. P. F.; GOMES, R. A. T. Aplicação do método de identificação espectral para imagens do sensor ASTER em ambiente de cerrado. Revista Brasileira de Geofísica, v. 23(2), p. 159-172, 2005.

CARVALHO Jr., O.A.; HERMUCHE, P.M.; GUIMARÃES, R.F. Identificação regional da floresta estacional decidual na bacia do Rio Paranã a partir da análise multitemporal de imagens MODIS. Revista Brasileira de Geofísica, v. 24(3), p. 319-332, 2006.

CARVALHO Jr., O. A.; SILVA, N. C.; CARVALHO, A. P. F.; COUTO Jr., A. F.; SILVA, C. R.; SHIMABUKURO, Y. E.; GUIMARÃES, R. F.; GOMES, R. A. T. Combining noise-adjusted principal components transform and median filter techniques for denoising Modis temporal signatures. Revista Brasileira de Geofísica, v. 30(2), p. 147-157, 2012.

CATTANI, C. E. V.; MERCANTE, E.; SOUZA, C. H. W.; WRUBLACK, S. C. Desempenho de algoritmos de classificação supervisionada para imagens dos satélites RapidEye. IN: Anais XVI Simpósio Brasileiro de Sensoriamento Remoto - SBSR, Foz do Iguaçu, PR, Brasil, 13 a 18 de abril de 2013, INPE.

CERQUEIRA, E. O.; POPPI, R. J.; KUBOTA, L. T. Utilização de filtro de transformada de Fourier para a minimização de ruídos em sinais analíticos. Química Nova, v. 23(5), 690-698.

CHEN, J.; JONSSON, P.; TAMURA, M.; GU, Z.; MATSUSHITA, B., EKLUNDH, L. A simple method for reconstructing a high-quality NDVI timeseries data set based on the Savitzky-Golay filter. Remote Sensing of Environment, v. 91, p. 332-344, 2004.

COUTO Jr., A. F.; CARVALHO Jr., O. A; MARTINS, E. S. Séries temporais de NDVI, EVI e NDWI do sensor MODIS para caracterização fenológica do algodão. Revista Brasileira de Cartografia, v. 65(1), p. 199-210, 2013.

COUTO Jr., A.F.; CARVALHO Jr., O.A.; MARTINS, E.S. Séries temporais MODIS aplicadas em sucessão de culturas de soja (Glycine max (L.) Merrill) e milho (Zea mays L.) em sistema de plantio direto. Revista Brasileira de Cartografia, v. 64, p. 405-418, 2012.

EKLUNDH, L.; JONSSON, P. Timesat 3.1. Software Manual. Lund, Suécia: Universidade do Lund, 2012, $82 \mathrm{p}$.

FLORES, P. M.; GUIMARÃES, R. F.; CARVALHO Jr., O. A.; GOMES, R. A. T. Análise multitemporal da expansão agrícola no município de Barreiras - Bahia (1988-2008). Campo-Território: Revista de Geografia Agrária, v. 7(14), p. 119, 2012.

FREITAS, R. M.; ARAI, E.; ADAMI, M.; SOUZA, A. F.; SATO, F. Y.; SHIMABUKURO, Y. E.; ROSA, R. R.; ANDERSON, L. O.; RUDORFF, B. F. T. Virtual laboratory of remote sensing series: visualization of MODIS EVI2 data set over South America. Journal of Computational Interdisciplinary Sciences, v. 2, p. 57-64. 2011. 
GALFORD, G. L.;MUSTARD, J. F.; MELILLO, J.; GENDRIN, A.; CERRI, C. C.; CERRI, C. E. P. Wavelet analysis of MODIS time series to detect expansion and intensification of row-crop agriculture in Brazil. Remote Sensing of Environment, v. 112, p. 576-587, 2008.

GREEN, A. A.; BERMAN, M.; SWITZER, B.; CRAIG, M. D. A transformation for ordering multispectral data in terms of image quality with implications for noise removal. IEEE Transactions on Geoscience and Remote Sensing, v. 26(1), p. 65-74, 1988.

HILL, M. Vegetation index suites as indicators of vegetation state in grassland and savanna: an analysis with simulated Sentinel 2 data for a North American transect. Remote Sensing of Environment, v. 137, p. 94-111, 2013.

HIRD, J. N.; McDERMID, G. J. Noise reduction of NDVI time-series: an empirical comparison of selected techniques. Remote Sensing of Environment, v.113, p. 248-258, 2009.

HUETE, A.; DIDAN, K.; MIURA, T.; RODRIGUEZ, E. P.; GAO, X.; FERREIRA, L. G. Overview of the radiometric and biophysical performance of the MODIS vegetation indices. Remote Sensing of Environment, v. 83(1-2), p. 195-213, 2002.

INGEBRITSEN, S. E.; LYON, R. J. P. Principal component analysis of multitemporal image pairs. International Journal of Remote Sensing, v. 6(5), p. 687-696, 1985.

JENSEN, J. R. Remote Sensing of the Environment: An Earth Resource Perspective. Upper Saddle River, NJ: Prentice Hall., $2^{\mathrm{a}}$ ed., 2006, 608 p.

JÖNSSON, P.; EKLUNDH, L. TIMESAT: a program for analyzing time-series of satellite sensor data. Computers \& Geosciences, v. 30(1), p. 833-845, 2004.

JUSTICE, C. O.; TOWNSHEND, J. R. G.; VERMOTE, E. F.; MASUOKA, E.; WOLFE, R. E.; SALEOUS, N.; ROY, D. P.; MORISETTE, J. T. An overview of MODIS land data processing and product status. Remote Sensing of Environment, v. 83, p. 3-15, 2002.

KRUSE, F. A.; LEFKOFF, A. B.; BOARDMAN, J. B.; HEIDEBRECHT, K. B.; SHAPIRO, A. T.; BARLOON, P. J.; GOETZ, A. F. H. The Spectral Image Processing Systems (SIPS) - interactive visualization and analysis of imaging spectrometer data. Remote Sensing of Environment, v. 44, p. 145-163, 1993.

LANDIS, J. R.; KOCH, G. G. The measurement of observer agreement for categorical data. Biometrics, v.33(1), p.159-174, 1977.

LEEUWEN, W. J. D.; HUETE, A. R.; LAING, T. W. MODIS vegetation index compositing approach: a prototype with AVHRR data. Remote Sensing of Environment, v. 69, p.264-280, 1999.

LU, X.; LIU, R.; LIU, J.; LIANG, S. Removal of noise by wavelet method to generate high quality temporal data of terrestrial MODIS products. Photogrammetric Engineering \& Remote Sensing, v. 73(10), p. 1-11, 2007.

MA, X.; HUETE, A.; YU, Q.; COUPE, N. R.; DAVIES, K.; BROICH, M.; RATANA, P.; BERINGER, J.; HUTLEY, L.B.; CLEVERLY, J.; BOULAIN, 
N.; EAMUS, D. Spatial patterns and temporal dynamics in savanna vegetation phenology across the North Australian tropical transect. Remote Sensing of Environment, v. 139, p. 97-115, 2013.

MENKE, A. B.; CARVALHO Jr., O. A.; GOMES, R. A. T.; MARTINS, E. S.; OLIVEIRA, S. N. Análise das mudanças do uso agrícola da terra a partir de dados de sensoriamento remoto multitemporal no município de Luis Eduardo Magalhães (BA - Brasil). Sociedade \& Natureza, v. 21(3), p. 315-326, 2009.

NOU, E. A. V.; COSTA, N. L. D. A. Diagnóstico da qualidade ambiental da bacia do rio São Francisco. Sub-bacias do Oeste Baiano e Sobradinho. Rio de Janeiro: IBGE, 111 p., 1994 (Série Estudos e Pesquisas em Geociências, 2.).

O'CONNOR, B.; DWYER, E.; CAWKWELL, F.; EKLUNDH, L. Spatio-temporal patterns in vegetation start of season across the island of Ireland using the MERIS global vegetation index. ISPRS Journal of Photogrammetry and Remote Sensing, v. 68, p. 79-94, 2012.

PIROMAL, R. A. S.; RIVERA-LOMBARDI, R. J.; SHIMABUKURO, Y. E.; FORMAGGIO, A. R.; KRUG, T. Utilização de dados MODIS para a detecção de queimadas na Amazônia. Acta Amazonica. v. 38(1), p. 77-84, 2008.

RIBEIRO, J. F.; WALTER, B. M. T. As principais fitofisionomias do Cerrado. In: SANO, S. M.; ALMEIDA, S. P.; RIBEIRO, J. F. (eds.), Cerrado. Ecologia e Flora. Planaltina, Embrapa Cerrados, v. 1, Cap. 6, p. 151-199, 2008.

ROSSO, P. H.; USTIN, S. L.; HASTINGS, A. Mapping marshland vegetation of San Francisco Bay, California, using hyperspectral data. International Journal of Remote Sensing. v. 26(23), p. 5169-5191, 2005.

ROWAN, L. C.; MARS, J. C. Lithologic mapping in the Mountain Pass, California area using Advanced Spaceborne Thermal Emission and Reflection Radiometer (ASTER) data. Remote Sensing of Environment, v. 84, p.350-366, 2003.

SANO, E. E.; FERREIRA, L. G.; ASNER, G. P.; STEINKE, E. T. Spatial and temporal probabilities of obtaining cloud-free Landsat images over the Brazilian tropical savana. International Journal of Remote Sensing, v. 28(12), p. 2739-2752. 2007.

SANO, E. E.; SANTOS, E. M.; MENESES, P. R. Análise de imagens do satélite Alos Palsar para o mapeamento de uso e cobertura da terra do Distrito Federal. Geociências, v. 28(4), p. 441-451, 2009.

SANO, E. E.; SANTOS, C. C. M.; SILVA, E. M.; CHAVES, J. M. Fronteira agrícola do oeste baiano: considerações sobre os aspectos temporais e ambientais. Geociências, v. 30(3), p. 479-489, 2011.

SANTOS, C. C. M.; VALE, R. M. C.; LOBÃO, J. S. B. Modernização da agricultura e ocupação de cerrados no oeste baiano. In: Oeste da Bahia: Trilhando velhos e novos caminhos do Além São Francisco. SANTOS, C. C. M.; VALE, R. M. C. (Org.). UEFS Editora, p.175-226, 2012. 
SEI. SUPERINTENDÊNCIA DE ESTUDOS ECONÔMICOS E SOCIAIS DA BAHIA. Balanço hídrico do estado da Bahia (Série Estudos e Pesquisas, 45). Salvador: SEI, 250 p., 1999.

SOARES, J. V.; BATISTA, G. T.; SHIMABUKURO, Y. E. Histórico e descrição. In: RUDORFF, B. F. T.; SHIMABUKURO, Y. E.; CEBALLOS, J. C. (Org.). $O$ Sensor Modis e suas Aplicações Ambientais no Brasil. São José dos Campos: Parêntese, 2007, p. 3-22.

SOUTH, S.; QI, J.; LUSCH, D. P. Optimal classification methods for mapping agricultural tillage practices. Remote Sensing of Environment. v. 91, p. 90-97, 2004.

TUCKER, C. J. Red and photographic infrared linear combinations for monitoring vegetation. Remote Sensing of Environment, v. 8, p. 127-150, 1979.

TYC, G.; TULIP, J.; SCHULTEN, D.; KRISCHKE, M.; OXFORT, M. The RapidEye mission design. Acta Astronautica, v. 56, p. 213-219, 2005.

VASCONCELOS, V.; CARVALHO Jr., O. A.; MARTINS, E. S.; COUTO Jr., A. F.; GUIMARÃES, R. F.; GOMES, R. A. T. Sistema de classificação geomorfométrica baseado em uma arquitetura sequencial em duas etapas: árvore de decisão e classificador espectral, no Parque Nacional Serra da Canastra. Revista Brasileira de Geomorfologia, v.13(2), p. 171-186, 2012.

WARDLOW, B. D.; EGBERT, S. L.; KASTENS, J. H. Analysis of time-series MODIS $250 \mathrm{~m}$ vegetation index data for crop classification in the U.S. Central Great Plains. Remote Sensing of Environment, v. 108, p. 290-310, 2007.

(Recebido em outubro de 2013. Aceito em abril de 2014). 